\title{
Vortex-type Half-BPS Solitons in ABJM Theory
}

\author{
Chanju Kim \\ Institute for the Early Universe and Department of Physics \\ Ewha Womans University, Seoul 120-750, Korea \\ and \\ Korea Institute for Advanced Study, 130-722, Korea \\ cjkim@ewha.ac.kr \\ Yoonbai Kim, O-Kab Kwon, Hiroaki Nakajima \\ Department of Physics, BK21 Physics Research Division, and Institute of Basic Science \\ Sungkyunkwan University, Suwon 440-746, Korea \\ yoonbai, okab, nakajima@skku.edu
}

\begin{abstract}
We study Aharony-Bergman-Jafferis-Maldacena (ABJM) theory without and with mass deformation. It is shown that maximally supersymmetry preserving, D-term, and F-term mass deformations of single mass parameter are equivalent. We obtain vortex-type halfBPS equations and the corresponding energy bound. For the undeformed ABJM theory, the resulting half-BPS equation is the same as that in supersymmetric Yang-Mills theory and no finite energy regular BPS solution is found. For the mass-deformed ABJM theory, the halfBPS equations for $\mathrm{U}(2) \times \mathrm{U}(2)$ case reduce to the vortex equation in Maxwell-Higgs theory, which supports static regular multi-vortex solutions. In $\mathrm{U}(N) \times \mathrm{U}(N)$ case with $N>2$ the nonabelian vortex equation of Yang-Mills-Higgs theory is obtained.
\end{abstract}




\section{Introduction}

The low-energy limit of M-theory is 11-dimensional supergravity and involves membranes and five-branes as solitonic extended objects [1]. Recently, the Bagger-Lambert-Gustavsson (BLG) theory [2, 3] and the Aharony-Bergman-Jafferis-Maldacena (ABJM) theory [4] have been proposed as the low-energy limit of world-volume theory of multiple M2-branes. The ABJM theory is given in the basis of brane constructions and is described by (1+2)-dimensional Chern-Simons-matter theories with $\mathrm{U}(N) \times \mathrm{U}(N)$ or $\mathrm{SU}(N) \times \mathrm{SU}(N)$ gauge group and $\mathcal{N}=6$ supersymmetry (SUSY). In large $N$ limit, the ABJM theory is dual to M-theory on $\mathrm{AdS}_{4} \times \mathrm{S}^{7} / \mathbb{Z}_{k}$, where $k$ is related with the discrete level of Chern-Simons term.

When the world-volume theory of the stacked M2-branes is constructed, one of the main tests is to reproduce Basu-Harvey fuzzy funnel [5] as a BPS configuration [2]. Along this line, the composite of M2-M5 and the domain wall solutions are studied in the BLG theory [6, 7, 8, and the ABJM theory [9] without and with mass deformation. When these codimension-one BPS objects are dealt, role of the two Chern-Simons gauge fields is completely missing. Among diverse research directions in the world-volume theory of M2-branes, it deserves to investigate BPS solitons for which the gauge fields play a crucial role. These are nothing but point-like BPS Chern-Simons vortices.

Relativistic Chern-Simons-Higgs theory with sextic scalar potential is first introduced in order to saturate the BPS bound for the static multi-BPS vortex solutions [10], and also arises in the supersymmetric abelian Chern-Simons-Higgs theories [11]. An attractive point of the BPS limit of Chern-Simons-Higgs theories is rich soliton spectrum due to coexistence of both the symmetric and broken vacua. In addition to the topologically stable multi-BPS vortices and domain walls, marginally stable nontopological solitons (or Q-balls) and nontopological vortices (or Q-vortices) exist [12]. Extension to $\mathrm{U}(1) \times \mathrm{U}(1)$ gauge group [13] and nonabelian gauge group [14] is also made. Therefore, in the scheme of $(1+2)$-dimensional quantum field theories, the mass-deformed BLG and ABJM theories are understood as the complicated Chern-Simons-Higgs theories, and the undeformed BLG and ABJM theories as their superconformal limit.

In this paper, we first discuss relation among the proposed mass deformations in the ABJM theory, with single mass deformation parameter. One is maximally supersymmetric mass deformation in terms of $\mathcal{N}=1$ superfield formalism [6] and in component fields [15]. Two other types of mass deformation correspond to D-term and F-term deformations in the basis of $\mathcal{N}=2 \mathrm{su}-$ perfield formalism [15]. Though they look different theories possessing different manifest SUSY's, we shall show that the aforementioned three mass deformations are equivalent. The main subject of our interest is to understand static vortex-type half-BPS objects of the ABJM theory without and with mass deformation. In BLG theory of $\mathrm{SU}(2) \times \mathrm{SU}(2)$ gauge symmetry, possible BPS equations were classified and some vortex-type BPS configurations were obtained [16, 17, 18]. In ABJM theory, some Chern-Simons vortex-type $\frac{1}{6}$-BPS solitons were obtained, including topological vortices, nontopological solitons, and nontopological vortices [19], and vortex loop operators 
were constructed [20]. Here we examine half-BPS equations for static vortex-type solitons in the $\mathrm{U}(N) \times \mathrm{U}(N)$ ABJM theory both without and with mass deformation, and discuss in detail the possible singular and regular multi-BPS vortex solutions. Though various point-like solitons are obtained in the world-volume theory of M2-branes as singular solutions without mass deformation and regular solutions with mass deformation, they await proper interpretation in the context of M-theory.

The rest of this paper is organized as follows. We begin section 2 with introduction of the ABJM theory, and in subsection 2.1 we discuss the relation among three proposed mass deformations. In section 3 vortex-type half BPS equations and the corresponding energy bound are obtained. In section 4 we reduce the general half-BPS equations in undeformed theory in a simple set of two coupled first-order equations which is the same as that in supersymmetric Yang-Mills theory with the Yang-Mills coupling identified as what obtained in D2 limit of the theory [21, 22]. There is no static vortex-like half-BPS solution with finite energy. In section 5, we examine half-BPS equations in mass-deformed theory. We first consider $\mathrm{U}(2) \times \mathrm{U}(2)$ case, leading to the vortex equation in Maxwell-Higgs theory, and find spinless multi-BPS vortex vortices without or with constant background magnetic field. For $\mathrm{U}(N) \times \mathrm{U}(N)$ case with $N>2$, under a suitable ansatz, the BPS equations reduce to the nonabelian vortex equation in Yang-Mills-Higgs theory. We also obtain other equations with different ansatz. We conclude in section 6 with brief summary and discussion.

\section{ABJM Theory with and without Mass Deformation}

The ABJM theory is an $\mathcal{N}=6$ superconformal $\mathrm{U}(N) \times \mathrm{U}(N)$ Chern-Simons theory with level $(k,-k)$ coupled to four complex scalars and four Dirac fermions in the bifundamental representation,

$$
\begin{gathered}
S_{\mathrm{ABJM}}=\int d^{3} x\left\{\frac{k}{4 \pi} \epsilon^{\mu \nu \lambda} \operatorname{tr}\left(A_{\mu} \partial_{\nu} A_{\lambda}+\frac{2 i}{3} A_{\mu} A_{\nu} A_{\lambda}-\hat{A}_{\mu} \partial_{\nu} \hat{A}_{\lambda}-\frac{2 i}{3} \hat{A}_{\mu} \hat{A}_{\nu} \hat{A}_{\lambda}\right)\right. \\
\left.-\operatorname{tr}\left(D_{\mu} Y_{A}^{\dagger} D^{\mu} Y^{A}\right)+\operatorname{tr}\left(\psi^{A \dagger} i \gamma^{\mu} D_{\mu} \psi_{A}\right)-V_{\text {ferm }}-V_{0}\right\}
\end{gathered}
$$

where $A=1, \ldots, 4$ and

$$
D_{\mu} Y^{A}=\partial_{\mu} Y^{A}+i A_{\mu} Y^{A}-i Y^{A} \hat{A}_{\mu}
$$

$V_{\text {ferm }}$ is the Yukawa-type quartic-interaction term,

$$
\begin{gathered}
V_{\mathrm{ferm}}=\frac{2 i \pi}{k} \operatorname{tr}\left(Y_{A}^{\dagger} Y^{A} \psi^{B \dagger} \psi_{B}-Y^{A} Y_{A}^{\dagger} \psi_{B} \psi^{B \dagger}+2 Y^{A} Y_{B}^{\dagger} \psi_{A} \psi^{B \dagger}-2 Y_{A}^{\dagger} Y^{B} \psi^{A \dagger} \psi_{B}\right. \\
\left.-\epsilon^{A B C D} Y_{A}^{\dagger} \psi_{B} Y_{C}^{\dagger} \psi_{D}+\epsilon_{A B C D} Y^{A} \psi^{B \dagger} Y^{C} \psi^{D \dagger}\right)
\end{gathered}
$$


and $V_{0}$ is the sextic scalar potential,

$$
\begin{aligned}
V_{0}=-\frac{4 \pi^{2}}{3 k^{2}} \operatorname{tr} & Y^{A} Y_{A}^{\dagger} Y^{B} Y_{B}^{\dagger} Y^{C} Y_{C}^{\dagger}+Y_{A}^{\dagger} Y^{A} Y_{B}^{\dagger} Y^{B} Y_{C}^{\dagger} Y^{C} \\
& \left.+4 Y^{A} Y_{B}^{\dagger} Y^{C} Y_{A}^{\dagger} Y^{B} Y_{C}^{\dagger}-6 Y^{A} Y_{B}^{\dagger} Y^{B} Y_{A}^{\dagger} Y^{C} Y_{C}^{\dagger}\right)
\end{aligned}
$$

We choose real gamma matrices with the convention $\gamma^{2}=\gamma^{0} \gamma^{1}$. An explicit representation would be

$$
\gamma^{0}=i \sigma^{2}, \quad \gamma^{1}=\sigma^{1}, \quad \gamma^{2}=\sigma^{3}
$$

The action (2.1) is known to be invariant under the $\mathcal{N}=6$ supersymmetry transformation 4 , 6. 23, 9],

$$
\begin{aligned}
\delta Y^{A} & =i \omega^{A B} \psi_{B}, \\
\delta \psi_{A} & =-\gamma_{\mu} \omega_{A B} D_{\mu} Y^{B}+\frac{2 \pi}{k}\left[-\omega_{A B}\left(Y^{C} Y_{C}^{\dagger} Y^{B}-Y^{B} Y_{C}^{\dagger} Y^{C}\right)+2 \omega_{B C} Y^{B} Y_{A}^{\dagger} Y^{C}\right] \\
& =-\gamma^{\mu} \omega_{A B} D_{\mu} Y^{B}+\omega_{B C}\left(\beta_{A}^{B C}+\delta_{A}^{[B} \beta_{D}^{C] D}\right), \\
\delta A_{\mu} & =-\frac{2 \pi}{k}\left(Y^{A} \psi^{B \dagger} \gamma_{\mu} \omega_{A B}+\omega^{A B} \gamma_{\mu} \psi_{A} Y_{B}^{\dagger}\right), \\
\delta \hat{A}_{\mu} & =\frac{2 \pi}{k}\left(\psi^{A \dagger} Y^{B} \gamma_{\mu} \omega_{A B}+\omega^{A B} \gamma_{\mu} Y_{A}^{\dagger} \psi_{B}\right),
\end{aligned}
$$

where $\omega_{A B}$ are supersymmetry transformation parameters with

$$
\omega^{A B}=\left(\omega_{A B}\right)^{*}=-\frac{1}{2} \epsilon^{A B C D} \omega_{C D}
$$

and

$$
\beta_{C}^{A B}=\frac{4 \pi}{k} Y^{[A} Y_{C}^{\dagger} Y^{B]}
$$

The form of the potential (2.4) is manifestly $\mathrm{SU}(4)$ invariant but is not manifestly positivedefinite. It can be written in a positive-definite form [24, 25] using the combination appearing in the second term of $\delta \psi_{A}$,

$$
V_{0}=\frac{2}{3}\left|\beta_{A}^{B C}+\delta_{A}^{[B} \beta_{D}^{C] D}\right|^{2}
$$

where, for convenience, we have introduced the notation $|\mathcal{O}|^{2} \equiv \operatorname{tr} \mathcal{O}^{\dagger} \mathcal{O}$.

There exists a unique mass deformation of the ABJM theory which respects the full $\mathcal{N}=6$ supersymmetry [6]. For the mass-deformed theory, the supersymmetric transformations (2.6) remain unchanged except the fermion fields for which there is an additional transformation,

$$
\delta_{\mathrm{m}} \psi_{A}=\mu M_{A}^{B} \omega_{B C} Y^{C}
$$


where $\mu$ is the mass deformation parameter and $M_{A}^{B}=\operatorname{diag}(1,1,-1,-1)$. This reduces the $\mathrm{R}$ symmetry from $\mathrm{SU}(4)$ to $\mathrm{SU}(2) \times \mathrm{SU}(2) \times \mathrm{U}(1)$, and it leads to the following additional terms to the Lagrangian,

$$
\begin{aligned}
\Delta V_{\text {ferm }} & =\operatorname{tr} \mu \psi^{\dagger A} M_{A}^{B} \psi_{B}, \\
\Delta V_{0} & =\operatorname{tr}\left(\frac{4 \pi \mu}{k} Y^{A} Y_{A}^{\dagger} Y^{B} M_{B}^{C} Y_{C}^{\dagger}-\frac{4 \pi \mu}{k} Y_{A}^{\dagger} Y^{A} Y_{B}^{\dagger} M_{C}^{B} Y^{C}+\mu^{2} Y_{A}^{\dagger} Y^{A}\right) .
\end{aligned}
$$

Combined with (2.9), the potential $V_{\mathrm{m}}$ in the mass-deformed theory can also be written in a manifestly positive-definite form,

$$
V_{\mathrm{m}}=V_{0}+\Delta V_{0}=\frac{2}{3}\left|\beta_{A}^{B C}+\delta_{A}^{[B} \beta_{D}^{C] D}+\mu M_{A}^{[B} Y^{C]}\right|^{2}
$$

This form is suitable for obtaining the half-BPS equations in the next section.

\subsection{Other formulations of mass-deformed theory}

The ABJM theory can be described in terms of either the formalism of the component fields as above, $\mathcal{N}=1$ superfields or $\mathcal{N}=2$ superfields. Depending on the formalism used, part of the symmetry is hidden and the resulting forms of the potential look quite different from each other.

This is also true for the mass-deformed theory. The maximally supersymmetric mass-deformed theory given by (2.10) and (2.11) was first examined in terms of $\mathcal{N}=1$ superfield formalism [26] and in component fields [15]. In addition to this mass deformation, two other types of mass deformation have been proposed in $\mathcal{N}=2$ superfield formalism in [15]. They correspond to a D-term deformation and an F-term deformation respectively and seem to produce different theories having only $\mathcal{N}=2$ supersymmetry. There is however a possibility that they have hidden symmetries not manifest in $\mathcal{N}=2$ formalism and may actually result in the same theory. Here we show that this is indeed the case. In other words, mass-deformed theories obtained by deforming D-term or F-term in $\mathcal{N}=2$ superfield formalism are the same as the one considered above with maximal $\mathcal{N}=6$ supersymmetry.

Let us first start with $\mathcal{N}=1$ formalism. Introducing the notation $Y^{A}=\left(Z^{1}, Z^{2}, W^{\dagger 1}, W^{\dagger 2}\right)$, $\mathcal{N}=1$ superpotential of ABJM theory is given as

$$
\begin{array}{r}
\mathcal{W}_{\mathcal{N}=1}=\frac{2 \pi}{k} \operatorname{tr}\left(\frac{1}{2} Z_{a}^{\dagger} Z^{a} Z_{b}^{\dagger} Z^{b}-\frac{1}{2} Z^{a} Z_{a}^{\dagger} Z^{b} Z_{b}^{\dagger}+\frac{1}{2} W_{a} W^{\dagger a} W_{b} W^{\dagger b}-\frac{1}{2} W^{\dagger a} W_{a} W^{\dagger b} W_{b}\right. \\
\left.+Z^{a} Z_{a}^{\dagger} W^{\dagger b} W_{b}-Z_{a}^{\dagger} Z^{a} W_{b} W^{\dagger b}+2 Z_{a}^{\dagger} Z^{b} W_{b} W^{\dagger a}-2 Z^{a} Z_{b}^{\dagger} W^{\dagger b} W_{a}\right),
\end{array}
$$

where $a, b=1,2$. The bosonic potential can be written in the perfect square form

$$
V_{0}=\operatorname{tr}\left(\hat{N}_{a}^{\dagger} \hat{N}^{a}+\hat{M}^{\dagger a} \hat{M}_{a}\right)
$$


with

$$
\begin{aligned}
\hat{N}^{a} & =-\frac{\partial \mathcal{W}_{\mathcal{N}=1}}{\partial Z_{a}^{\dagger}} \\
& =\frac{2 \pi}{k}\left(Z^{b} Z_{b}^{\dagger} Z^{a}-Z^{a} Z_{b}^{\dagger} Z^{b}-W^{\dagger b} W_{b} Z^{a}+Z^{a} W_{b} W^{\dagger b}-2 Z^{b} W_{b} W^{\dagger a}+2 W^{\dagger a} W_{b} Z^{b}\right), \\
\hat{M}_{a} & =\frac{\partial \mathcal{W}_{\mathcal{N}=1}}{\partial W^{\dagger a}} \\
& =\frac{2 \pi}{k}\left(W_{b} W^{\dagger b} W_{a}-W_{a} W^{\dagger b} W_{b}+W_{a} Z^{b} Z_{b}^{\dagger}-Z_{b}^{\dagger} Z^{b} W_{a}+2 Z_{a}^{\dagger} Z^{b} W_{b}-2 W_{b} Z^{b} Z_{a}^{\dagger}\right) .
\end{aligned}
$$

The SUSY-preserving mass deformation is introduced by the additional $\mathcal{N}=1$ superpotential as

$$
\Delta \mathcal{W}_{\mathcal{N}=1}=-\mu \operatorname{tr}\left(Z_{a}^{\dagger} Z^{a}-W^{\dagger a} W_{a}\right)
$$

which corresponds to the following replacement in the bosonic potential (2.14)

$$
\hat{N}^{a} \rightarrow \hat{N}^{a}+\mu Z^{a}, \quad \hat{M}_{a} \rightarrow \hat{M}_{a}+\mu W_{a} .
$$

Then the potential in the deformed theory is

$$
V_{\mathrm{m}}=\left|\hat{N}^{a}+\mu Z^{a}\right|^{2}+\left|\hat{M}_{a}+\mu W_{a}\right|^{2}
$$

which can be shown to be the same as (2.9).

In terms of $\mathcal{N}=2$ superfield formalism, the bosonic potential of ABJM theory is written as the sum of the D-term potential $V_{D}$ and the F-term potential $V_{F}$ [27]

$$
V_{0}=V_{D}+V_{F}
$$

where

$$
\begin{aligned}
V_{D} & =\operatorname{tr}\left(N_{a}^{\dagger} N^{a}+M^{\dagger a} M_{a}\right), \\
N^{a} & =\frac{2 \pi}{k}\left(Z^{b} Z_{b}^{\dagger} Z^{a}-Z^{a} Z_{b}^{\dagger} Z^{b}-W^{\dagger b} W_{b} Z^{a}+Z^{a} W_{b} W^{\dagger b}\right), \\
M_{a} & =\frac{2 \pi}{k}\left(W_{b} W^{\dagger b} W_{a}-W_{a} W^{\dagger b} W_{b}+W_{a} Z^{b} Z_{b}^{\dagger}-Z_{b}^{\dagger} Z^{b} W_{a}\right), \\
V_{F} & =\operatorname{tr}\left(F_{a}^{\dagger} F^{a}+G^{\dagger a} G_{a}\right), \\
F^{a} & =\frac{4 \pi}{k} \epsilon^{a c} \epsilon_{b d} W^{\dagger b} Z_{c}^{\dagger} W^{\dagger d}, \\
G_{a} & =-\frac{4 \pi}{k} \epsilon_{a c} \epsilon^{b d} Z_{b}^{\dagger} W^{\dagger c} Z_{d}^{\dagger} .
\end{aligned}
$$

The F-term potential $V_{F}$ is obtained from the $\mathcal{N}=2$ superpotential $\mathcal{W}_{\mathcal{N}=2}$ as

$$
\begin{gathered}
\mathcal{W}_{\mathcal{N}=2}=\frac{2 \pi}{k} \epsilon_{a c} \epsilon^{b d} \operatorname{tr}\left(Z^{a} W_{b} Z^{c} W_{d}\right) \\
F^{a}=\frac{\partial \mathcal{W}_{\mathcal{N}=2}^{\dagger}}{\partial Z_{a}^{\dagger}}, \quad G_{a}=\frac{\partial \mathcal{W}_{\mathcal{N}=2}^{\dagger}}{\partial W^{\dagger a}}
\end{gathered}
$$


In $\mathcal{N}=2$ superfield formalism, we can consider two kinds of mass deformations, D-term deformation and F-term deformation [15]. The D-term deformation is introduced by a replacement in (2.20)

$$
N^{a} \rightarrow N^{a}+\mu Z^{a}, \quad M_{a} \rightarrow M_{a}+\mu W_{a} .
$$

Nevertheless one can explicitly verify that the resulting potential is the same as (2.19), viz.,

$$
\left|N^{a}+\mu Z^{a}\right|^{2}+\left|M_{a}+\mu W_{a}\right|^{2}+\left|F^{a}\right|^{2}+\left|G_{a}\right|^{2}=\left|\hat{N}^{a}+\mu Z^{a}\right|^{2}+\left|\hat{M}_{a}+\mu W_{a}\right|^{2} .
$$

Fermionic part can also be shown to be identical. Hence the D-term deformation is the same as the maximally SUSY-preserving mass deformation. We note that the D-term deformation can be regarded as the Fayet-Illiopoulos term deformation when the gauge group is $\mathrm{U}(N) \times \mathrm{U}(N)$ (not $\mathrm{SU}(N) \times \mathrm{SU}(N))[15]$.

The other mass deformation is an F-term deformation which is introduced by the additional $\mathcal{N}=2$ superpotential

$$
\Delta \mathcal{W}_{\mathcal{N}=2}=\mu \operatorname{tr}\left(Z^{a} W_{a}\right)
$$

The deformation of bosonic potential is the form of (2.11) with the off-diagonal mass matrix

$$
M_{A}{ }^{B}=\left(\begin{array}{cccc}
0 & 0 & 1 & 0 \\
0 & 0 & 0 & 1 \\
1 & 0 & 0 & 0 \\
0 & 1 & 0 & 0
\end{array}\right) .
$$

By a field redefinition, this $M_{A}^{B}$ can be diagonalized and the F-term deformation is equivalent to the other deformations considered above. In particular, $\mathcal{N}=6$ supersymmetry is still preserved in every case by deforming the transformation law as (2.10). At first sight, the F-term deformation looks different from the other deformations since they have $\mathrm{SU}(2) \times \mathrm{SU}(2) \times \mathrm{U}(1)$, while in the case of the F-term deformation only diagonal $\mathrm{SU}(2)$ can be seen. However we can find the extra $\mathrm{SU}(2)$ and $\mathrm{U}(1)$ symmetries in the F-term deformation. From the form of the mass matrix (2.32), the generator of the extra $\mathrm{SU}(2)$ is obtained as

$$
\left(\begin{array}{cc}
0 & \frac{1}{2} \alpha_{i} \tau_{i} \\
\frac{1}{2} \alpha_{i} \tau_{i} & 0
\end{array}\right),
$$

and also the $\mathrm{U}(1)$ symmetry is generated by (2.32) itself. Here $\alpha_{i}(i=1,2,3)$ are the parameters of the extra $\mathrm{SU}(2)$ and $\tau_{i}$ are the Pauli matrices. Since both symmetries are generated by the off-diagonal matrices which mix $Z^{A}$ and $W^{\dagger A}$, their symmetries do not respect the structure of $\mathcal{N}=2$ superfield formalism (Recall that $Z^{A}$ is the lowest component of the chiral superfield whereas $W^{\dagger A}$ is the lowest component of the anti-chiral superfield). That is the reason why we can see only diagonal $\mathrm{SU}(2)$ in the F-term deformation. The fermionic mass term is also invariant under these extra $\mathrm{SU}(2)$ and $\mathrm{U}(1)$. 
Now we briefly discuss the vacua of the mass-deformed theory. From (2.12) the vacuum equation is given by

$$
\beta_{A}^{B C}+\delta_{A}^{[B} \beta_{D}^{C] D}+\mu M_{A}^{[B} Y^{C]}=0 .
$$

Contracting with $\delta_{C}^{A}$ yields $\beta_{D}^{B D}=\mu M_{D}^{B} Y^{D}$. Inserting this into (2.34), we obtain

$$
\beta_{A}^{B C}+\mu\left(\delta_{A}^{[B} M_{D}^{C]} Y^{D}+M_{A}^{[B} Y^{C]}\right)=0
$$

More explicitly, we have

$$
\begin{aligned}
& \beta_{a}^{a b}+\mu Y^{b}=0, \\
& \beta_{p}^{p q}-\mu Y^{q}=0, \\
& \beta_{a}^{b p}=\beta_{p}^{q a}=\beta_{a}^{p q}=\beta_{p}^{a b}=0,
\end{aligned}
$$

where $a, b=1,2$ and $p, q=3,4$. Equations (2.36) -(2.37) have been conjectured and analyzed in [15]. Since (2.36) reduces to (2.37) with the substitution $Y^{a} \rightarrow Y_{p}^{\dagger}$, we consider only (2.37) to which there is essentially a unique irreducible solution,

$$
Y_{m n}^{3}=\delta_{m n} \sqrt{\frac{k \mu}{2 \pi}} \sqrt{m-1}, \quad Y_{m n}^{4}=\delta_{m+1, n} \sqrt{\frac{k \mu}{2 \pi}} \sqrt{N-n} .
$$

Then from (2.38) we see that $Y^{1}=Y^{2}=0$ identically as claimed in [15].1]

\section{Half-BPS Equations}

Here we will obtain vortex-type half BPS equations in ABJM theory with and without mass deformation. First we consider the supersymmetric variation of the fermions $\delta \psi^{A}=0$ to obtain the BPS equations. Then we will get the energy bound by rewriting the energy functional in the form of complete squares.

We impose the supersymmetric condition of the form

$$
\gamma^{0} \omega_{A B}=i s_{A B} \omega_{A B}, \quad s_{A B}=s_{B A}= \pm 1
$$

which reduces the number of supersymmetries by half. Because of the property (2.7) among $\omega_{A B}$ 's, we should have $s_{34}=-s_{12}, s_{24}=-s_{13}$ and $s_{23}=-s_{14}$. With the help of $\gamma^{2}=-\gamma^{1} \gamma^{0}$, the supersymmetric variation of the fermion $\delta \psi_{A}$ can be reshuffled to

$$
\gamma^{0} \delta \psi_{A}=\left[\delta_{A}^{[B} D_{0} Y^{C]}+\gamma^{0}\left(\beta_{A}^{B C}+\delta_{A}^{[B} \beta_{D}^{C] D}+\mu M_{A}^{[B} Y^{C]}\right)\right] \omega_{B C}-\gamma^{2}\left(D_{1} Y^{B}-\gamma^{0} D_{2} Y^{B}\right) \omega_{A B},
$$

\footnotetext{
${ }^{1}$ We heard that the same result was also obtained by [28].
} 
where mass-deformed term (2.10) has been included. Then $\delta \psi_{A}=0$ implies that

$$
\begin{aligned}
& \left(D_{1}-i s_{A B} D_{2}\right) Y^{B}=0, \\
& \delta_{A}^{[B} D_{0} Y^{C]}+i s_{B C}\left(\beta_{A}^{B C}+\delta_{A}^{[B} \beta_{D}^{C] D}+\mu M_{A}^{[B} Y^{C]}\right)=0, \quad(\text { no sum over } B, C) .
\end{aligned}
$$

For nontrivial configurations at least one of $D_{i} Y^{A}$ should be nonzero. Assume $D_{1} Y^{1} \neq 0$ for definiteness. Then from the first equation with $A=1$, it immediately follows that

$$
s_{12}=s_{13}=s_{14} \equiv s
$$

should be the same and

$$
\left(D_{1}-i s D_{2}\right) Y^{1}=0 .
$$

For $A \neq 1$, since $s_{23}=s_{34}=s_{42}=-s$,

$$
\begin{array}{ll}
\left(D_{1}-i s D_{2}\right) Y^{A}=0 & (A \neq 1), \\
\left(D_{1}+i s D_{2}\right) Y^{A}=0 & (A \neq 1),
\end{array}
$$

where the first equation comes from $s_{A 1}=s$ and the second from $s_{A B}=-s$ for $A, B \neq 1$. Then

$$
D_{i} Y^{A}=0, \quad(A \neq 1)
$$

and hence only one field can be nontrivial in half-BPS configurations. This has also been obtained in [20, 29].

Similarly, from the second line of (3.3), we see that there are three different equations for each $D_{0} Y^{A}$. They will produce various constraints for consistency. Eventually we end up with following equations:

$$
\begin{array}{ll}
D_{0} Y^{1}+i s\left(\beta_{2}^{21}+\mu Y^{1}\right)=0, & D_{0} Y^{2}-i s\left(\beta_{1}^{12}+\mu Y^{2}\right)=0, \\
D_{0} Y^{3}-i s \beta_{1}^{13}=0, & D_{0} Y^{4}-i s \beta_{1}^{14}=0, \\
\beta_{3}^{31}=\beta_{4}^{41}=\beta_{2}^{21}+\mu Y^{1}, & \beta_{4}^{43}=\mu Y^{3}, \quad \beta_{3}^{34}=\mu Y^{4}, \\
\beta_{3}^{32}=\beta_{4}^{42}=\beta_{2}^{23}=\beta_{2}^{24}=0, & \\
\beta_{A}^{B C}=0 \quad(A \neq B \neq C \neq A) .
\end{array}
$$

Equations (3.5), (3.7), (3.8) form the full set of half-BPS equations. In addition, Gauss' laws should also be satisfied,

$$
\frac{k}{2 \pi} B=\frac{k}{2 \pi} F_{12}=j^{0}, \quad-\frac{k}{2 \pi} \hat{B}=-\frac{k}{2 \pi} \hat{F}_{12}=\hat{j}^{0}
$$

where $j^{0}$ and $\hat{j}^{0}$ are respectively charge densities of the conserved currents associated with $\mathrm{U}(1)$ rotations,

$$
\begin{aligned}
& j_{\mu}=i\left(Y^{A} D_{\mu} Y_{A}^{\dagger}-D_{\mu} Y^{A} Y_{A}^{\dagger}\right) \\
& \hat{j}_{\mu}=i\left(Y_{A}^{\dagger} D_{\mu} Y^{A}-D_{\mu} Y_{A}^{\dagger} Y^{A}\right) .
\end{aligned}
$$


The half-BPS equations can also be obtained from the bosonic part of the energy,

$$
E=\int d^{2} x\left(\left|D^{0} Y_{A}\right|^{2}+\left|D_{i} Y^{A}\right|^{2}+V_{\mathrm{m}}\right),
$$

where the potential $V_{\mathrm{m}}$ is given by (2.12). With the original form of the BPS equation (3.3) in mind, we can reshuffle the energy as

$$
\begin{aligned}
E= & \frac{1}{3} \int d^{2} x\left\{2 \sum_{A, B, C}\left|\delta_{A}^{[B} D_{0} Y^{C]}+i s_{B C}\left(\beta_{A}^{B C}+\delta_{A}^{[B} \beta_{D}^{C] D}+\mu M_{A}^{[B} Y^{C]}\right)\right|^{2}\right. \\
& \left.+\sum_{A \neq B}\left|\left(D_{1}-i s_{A B} D_{2}\right) Y^{A}\right|^{2}\right\} \\
& +i s \operatorname{tr} \int d^{2} x \epsilon_{i j} \partial_{i}\left(Y_{1}^{\dagger} D_{j} Y^{1}-\frac{1}{3} \sum_{A=2}^{4} Y_{A}^{\dagger} D_{j} Y^{A}\right)-\frac{s}{3} \mu \operatorname{tr} \int d^{2} x\left(j^{0}+2 J_{12}^{0}\right),
\end{aligned}
$$

where

$$
J_{12}^{0}=i\left(Y^{1} D_{0} Y_{1}^{\dagger}-D_{0} Y^{1} Y_{1}^{\dagger}\right)-i\left(Y^{2} D_{0} Y_{2}^{\dagger}-D_{0} Y^{2} Y_{2}^{\dagger}\right)
$$

is the charge density for an $\mathrm{SU}(4)$ rotation $Y^{1} \rightarrow e^{-i \alpha} Y^{1}, Y^{2} \rightarrow e^{i \alpha} Y^{2}$. In obtaining this expression we have used the Gauss' laws (3.9). Note that, for each and every $\mu$ and index $A,\left|D_{\mu} Y^{A}\right|^{2}$ is organized into three different complete squares in accordance with different supersymmetries and the factor $1 / 3$ in front of the integral accounts for the normalization.

The first two absolute-square terms in (3.12) precisely reproduce the half-BPS equations obtained before in (3.5), (3.7), and (3.8). The first term in the last line is a boundary term 2 which vanishes for well-behaved configurations. Then we get the energy bound

$$
E \geq \frac{1}{3}\left|\mu\left(Q+2 R_{12}\right)\right|
$$

where $Q=\operatorname{tr} \int d^{2} x j^{0}$ and $R_{12}=\operatorname{tr} \int d^{2} x J_{12}^{0}$. The energy bound which is saturated for any wellbehaved half-BPS configuration is proportional to the mass-deformation parameter $\mu$. Note that in the energy bound there is the overall $\mathrm{U}(1)$ charge $Q$ in addition to the R-charge $R_{12}$ which also exists in BLG case [6].

\section{Solving Half-BPS Equations without Mass Deformation}

Here we would like to solve half-BPS equations in the original ABJM theory $\mu=0$. In this case the equations are symmetric among $Y^{2}, Y^{3}, Y^{4}$.

\footnotetext{
${ }^{2}$ This bound can also be seen from the SUSY algebra obtained in [30].
} 
In the massless limit of $\mu \rightarrow 0$, the energy for half-BPS configurations is given by the total derivative term. From (3.12), we have

$$
E=\left|i \operatorname{tr} \int d^{2} x \epsilon_{i j} \partial_{i}\left(Y_{1}^{\dagger} D_{j} Y^{1}-\frac{1}{3} \sum_{A=2}^{4} Y_{A}^{\dagger} D_{j} Y^{A}\right)\right|
$$

which vanishes for every well-behaved field configuration. Therefore we expect that there would be no finite energy solution to the half-BPS equations other than vacuum configurations. Nevertheless one can consider solutions with infinite energy, which may be physically meaningful in the context of string theory.

The simplest solution would be obtained by assuming $Y^{2}=Y^{3}=Y^{4}=0$ for which the only remaining equation is (3.5). Magnetic fields vanish as $D_{0} Y^{A}=0$. Then $Y^{1}$ is an arbitrary (anti)holomorphic function which can be singular as $Y^{1} \sim z^{1 / k}$ at the origin. This solution has been discussed in the context of BLG theory with an M-theory interpretation [17].

To obtain more nontrivial solutions at least one of $Y^{2}, Y^{3}, Y^{4}$ should be nonzero. Due to the symmetry of the equations, we may assume $Y^{2} \neq 0$. Moreover with the help of the $\mathrm{U}(N) \times \mathrm{U}(N)$ gauge symmetry we can bring $Y^{2}$ to a diagonal form with increasing nonnegative real components,

$$
Y^{2}=\left(\begin{array}{llll}
v_{1} I_{n_{1}} & & & \\
& v_{2} I_{n_{2}} & & \\
& & \ddots & \\
& & & v_{k} I_{n_{k}}
\end{array}\right), \quad\left(0 \leq v_{1}<v_{2}<\cdots<v_{k}\right),
$$

where $\sum_{i=1}^{k} n_{i}=N$ and $I_{n_{i}}$ is the identity matrix of dimension $n_{i}$.

We first concentrate on the constraint equations in (3.8). From the equation $\beta_{2}^{23}=\beta_{2}^{24}=0$, it is not difficult to see that $Y^{3}$ and $Y^{4}$ are block diagonal,

$$
Y^{A}=\left(\begin{array}{cccc}
Y_{(1)}^{A} & & & \\
& Y_{(2)}^{A} & & \\
& & \ddots & \\
& & & Y_{(k)}^{A}
\end{array}\right), \quad(A=3,4)
$$

With these, $\beta_{3}^{24}=\beta_{2}^{43}=\beta_{3}^{32}=\beta_{4}^{42}=0$ gives

$$
\begin{array}{ll}
v_{i}\left[Y_{3(i)}^{\dagger}, Y_{(i)}^{4}\right]=0, & v_{i}\left[Y_{(i)}^{3}, Y_{(i)}^{4}\right]=0, \\
v_{i}\left[Y_{3(i)}^{\dagger}, Y_{(i)}^{3}\right]=0, & v_{i}\left[Y_{4(i)}^{\dagger}, Y_{(i)}^{4}\right]=0, \quad(\text { no sum over } i) .
\end{array}
$$

Note that all $v_{i}$ 's are positive possibly except $v_{1}$ which we assume nonzero for the moment. Then (4.4) implies that $Y^{3}$ and $Y^{4}$ are normal matrices and commute to each other and their conjugates. Therefore, under a suitable unitary transformation, $Y^{3}$ and $Y^{4}$ become completely diagonal. If $v_{1}=0$, we can utilize $\mathrm{U}\left(n_{1}\right) \times \mathrm{U}\left(n_{1}\right)$ symmetry to make $Y_{(1)}^{3}$ diagonal and reach the same conclusion. 
Now we apply the remaining constraints, namely $\beta_{A}^{1 B}=0$ where $A, B=2,3,4$ and $A \neq B$. Since $Y^{2}, Y^{3}, Y^{4}$ are all diagonal, this means $\left[Y^{1}, Y_{A}^{\dagger} Y^{B}\right]=0$. If all $Y_{A}^{\dagger} Y^{B}$ 's are nondegenerate, $Y^{1}$ must be diagonal. But then all scalar fields are diagonal and we will have only trivial solutions with vanishing magnetic field. To obtain nontrivial solutions, there should be a common subspace where all $Y_{A}^{\dagger} Y^{B}$ 's are degenerate. Moreover, it is not difficult to see that such a degenerate subspace should entirely belong to some degenerate subspace of $Y^{2}$ in (4.2). This in turn means that $Y^{1}$ can be at most block diagonal and in each block diagonal subspace $Y^{2}, Y^{3}, Y^{4}$ are all proportional to the identity.

It is now sufficient to work within each subspace where $Y^{A}=v^{A} I(A=2,3,4)$. From (3.7), we find that $v^{A}$ 's are constants and $A_{i}=\hat{A}_{i} . D_{0} Y^{A}$ 's are determined from (3.8),

$$
\begin{aligned}
& D_{0} Y^{1}=0 \\
& D_{0} Y^{A}=-i s \beta_{1}^{1 A}=i s \frac{2 \pi}{k} v^{A}\left[Y^{1}, Y_{1}^{\dagger}\right], \quad(A \neq 1) .
\end{aligned}
$$

Plugging this into the Gauss' laws (3.9), we are left with the following half-BPS equations without further constraint:

$$
\begin{aligned}
& \left(D_{1}-i s D_{2}\right) Y^{1}=0, \\
& B=\hat{B}=-\frac{s}{2}\left(\frac{2 \pi v}{k}\right)^{2}\left[Y^{1}, Y_{1}^{\dagger}\right]
\end{aligned}
$$

where $v^{2}=\sum_{A=2}^{4}\left|v^{A}\right|^{2}$ is a positive constant. Note that this result is completely general without any ansatz employed.

The equation (4.6) is not entirely new. It can be obtained as the half-BPS equation of the super Yang-Mills theory with coupling constant

$$
g_{\mathrm{YM}}=\frac{2 \pi v}{k}
$$

This identification has already appeared in the context of the compactification of BLG/ABJM theory (from M2 to D2) [21, 22]. For finite $k$, there are correction terms to Yang-Mills Lagrangian. However here we do not need to take the limit $v, k \rightarrow \infty$ as long as the half-BPS equation is concerned.

Alternatively, if the sign of the second equation in (4.6) is flipped, it is exactly the same as the half-BPS equation in nonrelativistic Chern-Simons theory with an adjoint matter [31, 32] where the solutions have been studied extensively. Here we briefly describe some simple solutions of (4.6) with $s=1$ for definiteness. Introducing complex notations $z=x_{1}+i x_{2}$ and $A=\left(A_{1}-i A_{2}\right) / 2$, we take the ansatz,

$$
\begin{aligned}
Y^{1} & =\sum_{a=1}^{N-1} y_{a} e^{a}+y_{M} E^{-M}, \\
A & =\sum_{a=1}^{N-1} A_{a} h^{a}, \quad \bar{A}=\sum_{a=1}^{N-1} A_{a}^{*} h^{a},
\end{aligned}
$$


where $h^{a}$ and $e^{a}$ are $\mathrm{SU}(N)$ generators in the Chevalley basis satisfying $\left[e^{a}, e^{-a^{\prime}}\right]=\delta_{a a^{\prime}} h^{a}$, $\left[h^{a}, e^{b}\right]=K_{a b} e^{b}$ with $K_{a b}$ being the Cartan matrix, and $E^{-M}$ is the Hermitian conjugate of the maximal ladder operator $E^{M}$ commuting with $e^{a}$ 's. Plugging (4.8) into (4.6), we obtain (affine-)Toda-type equation,

$$
\begin{aligned}
\partial \bar{\partial} \ln \left|y_{a}\right|^{2} & =4 v\left(\frac{2 \pi}{k}\right)^{2} \sum_{b=1}^{N-1} K_{a b}\left(\left|y_{b}\right|^{2}-\frac{|G(z)|^{2}}{\left|c_{b}\right|^{2} \prod_{c=1}^{N-1}\left|y_{c}\right|^{2}}\right), \\
y_{M} & =\frac{G(z)}{\prod_{a=1}^{N-1} y_{a}}
\end{aligned}
$$

where $G(z)$ is an arbitrary holomorphic function. For SU(2), this reduces to Liouville-type equation (with $G=0$ ) or Sinh-Gordon-type equation (with $G=$ const.) considered in [17] in the context of BLG theory. The solutions however all have to have infinite energy as we mentioned before. This is also consistent with the fact that (4.6) is obtained from super Yang-Mills theory without symmetry breaking potential.

\section{Solving Half-BPS Equations in the Mass-Deformed The- ory}

In this section we solve half-BPS equations in (3.5), (3.7), (3.8) in the mass-deformed theory. In this case, the constraint equations in (3.8) are more complicated and we first consider $\mathrm{U}(2) \times \mathrm{U}(2)$ case, and then discuss general $\mathrm{U}(N) \times \mathrm{U}(N)$ case.

\section{$5.1 \mathrm{U}(2) \times \mathrm{U}(2)$}

From the constraint $\beta_{4}^{43}=\mu Y^{3}$ and $\beta_{3}^{34}=\mu Y^{4}$, it is clear that $Y^{3}$ is nonzero if and only if $Y^{4}$ is nonzero. Let us first consider the case that both are nonzero. Utilizing the gauge symmetry, we may assume without loss of generality that $Y^{3}$ is diagonal with nonnegative real entries,

$$
Y^{3}=\sqrt{\frac{k|\mu|}{2 \pi}}\left(\begin{array}{ll}
c & 0 \\
0 & d
\end{array}\right), \quad 0 \leq c \leq d .
$$

Suppose $c \neq d$. Then solving the two constraints as well as $\beta_{A}^{B C}=0$, we find only a trivial solution that $Y^{1}=Y^{2}=0$ and 3

$$
Y^{3}=\sqrt{\frac{k \mu}{2 \pi}}\left(\begin{array}{ll}
0 & 0 \\
0 & d
\end{array}\right), \quad Y^{4}=\sqrt{\frac{k \mu}{2 \pi}}\left(\begin{array}{cc}
0 & 0 \\
e^{i \chi} & 0
\end{array}\right),
$$

\footnotetext{
${ }^{3}$ Hereafter we assume that $\mu>0$. Negative $\mu$ case can be analyzed in a similar manner and the same results are obtained except that the first and the second components are exchanged.
} 
where $\chi$ is a real constant.

To obtain nontrivial solutions $Y^{3}$ must be proportional to the identity. But then from the constraint $\mu Y^{4}=\beta_{3}^{34}$, we see that $Y^{4}=0$ which in turn implies $Y^{3}=0$. Therefore we are left with only one constraint,

$$
\beta_{2}^{21}+\mu Y^{1}=0 .
$$

Note that this constraint with the first BPS equation in (3.8) implies $D_{0} Y^{1}=0$ and hence only $Y^{2}$ is responsible for the charge of which the $\mathrm{U}(1)$ current is given in (3.10), while $Y^{1}$ satisfying (3.5) gives the vorticity. Nonzero charge due to $Y^{2}$ is then related to the magnetic field through the Gauss laws (3.9) which are a characteristic nature of the Chern-Simons gauge theory.

As before, we can assume that $Y^{2}$ is a diagonal matrix with nonnegative increasing real entries. Solving (5.3) gives

$$
Y^{1}=\sqrt{\frac{k \mu}{2 \pi}}\left(\begin{array}{ll}
0 & f \\
0 & 0
\end{array}\right), \quad Y^{2}=\sqrt{\frac{k \mu}{2 \pi}}\left(\begin{array}{cc}
a & 0 \\
0 & \sqrt{a^{2}+1}
\end{array}\right), \quad(a \geq 0) .
$$

Inserting this into the equation for $D_{0} Y^{2}$ in (3.8) and Gauss's laws (3.9), we find that the magnetic fields are given by

$$
B=\hat{B}=-2 s \mu^{2}\left(\begin{array}{cc}
a^{2}\left(1+|f|^{2}\right) & 0 \\
0 & \left(a^{2}+1\right)\left(1-|f|^{2}\right)
\end{array}\right),
$$

which means that the gauge fields are diagonal,

$$
A_{i}=\left(\begin{array}{cc}
u_{i} & 0 \\
0 & v_{i}
\end{array}\right) .
$$

Note that $\hat{A}_{i}$ is the same as $A_{i}$ up to a gauge degree which can be put to zero. Then from $D_{i} Y^{2}=0, a$ in (5.4) must be a constant.

Finally, from the equation $\left(D_{1}-i s D_{2}\right) Y^{1}=0$ in (3.5) , we can express the gauge fields in terms of $Y^{1}$. Explicitly, with $s=-1$ for definiteness,

$$
\bar{u}-\bar{v}=i \bar{\partial} \ln f .
$$

Comparing this with the magnetic field $B=\frac{2}{i}(\partial \bar{A}-\bar{\partial} A)$, we obtain

$$
\partial \bar{\partial} \ln |f|^{2}+i(\partial \bar{\partial}-\bar{\partial} \partial) \Omega=\mu^{2}\left[\left(2 a^{2}+1\right)|f|^{2}-1\right],
$$

where $\Omega$ is the phase of the scalar field, $f=|f| e^{i \Omega}$. This is the celebrated vortex equation appearing in Maxwell-Higgs theory and has been extensively studied [33]. The same equation has also obtained in mass-deformed BLG theory [18]. Note however that, although the final equation is the same, $\mathrm{U}(1)$ part plays a nontrivial role in the present case. 
Let us now calculate the energy of the solution satisfying (5.8). Since $D_{0} Y^{1}=0, J_{12}^{0}=j^{0}=$ $\frac{k}{2 \pi} B$ and hence

$$
E=\left|\frac{k \mu}{2 \pi} \operatorname{tr} \int d^{2} x B\right| .
$$

Therefore the energy is given by the trace part of the magnetic field while the vorticity of the solution comes from the relative part as seen in (5.7). Then the energy of a vortex solution is not proportional to the vorticity in general and can be infinite for some solutions. Using (5.5), we can rewrite

$$
E=\left|\frac{k \mu}{2 \pi} \int d^{2} x 2 \mu^{2}\left(2 a^{2}+1-|f|^{2}\right)\right| .
$$

This implies that, for finite energy solutions, $f$ should behave asymptotically as

$$
|f|^{2} \longrightarrow 2 a^{2}+1, \quad r \longrightarrow \infty,
$$

which is consistent with (5.8) only for $a=0$. In other words, to obtain finite energy solutions we should set $a=0$. In this case, $u_{i}=0$ and using (5.7), we find that the magnetic flux is given by

$$
\operatorname{tr} \int d^{2} x B=2 \pi n, \quad(n \in \mathbb{Z}),
$$

where $n$ is the vorticity. Then the energy becomes

$$
E=n k \mu \text {. }
$$

A characteristic nature of configurations in Chern-Simons gauge theory is that they usually carry nonzero angular momentum. However in this case it vanishes. To see this note that the linear momentum density is proportional to the combination $D_{0} Y^{A} D_{i} Y_{A}^{\dagger}$. However for the present case either $D_{0} Y^{A}$ vanishes or $D_{i} Y^{A}$ vanishes because fields do not carry both charge and vorticity as discussed above.

When $a \neq 0$, the solution may be interpreted as vortices in the presence of a constant magnetic field with the energy written as a sum of the vortex part and the constant part,

$$
E=\frac{n k \mu}{2 a^{2}+1}+\int d^{2} x \frac{4 k \mu^{3} a^{2}\left(a^{2}+1\right)}{\pi\left(2 a^{2}+1\right)} .
$$

It would be illuminating to examine the origin of the Maxwell-Higgs vortex equation in the Chern-Simons gauge theory which has a sextic potential having a minimum at the origin. For this purpose we consider the ansatz

$$
Y^{1}=\sqrt{\frac{k \mu}{2 \pi}}\left(\begin{array}{cc}
0 & f \\
0 & 0
\end{array}\right), \quad Y^{2}=\sqrt{\frac{k \mu}{2 \pi}}\left(\begin{array}{ll}
0 & 0 \\
0 & g
\end{array}\right), \quad Y^{3}=Y^{4}=0,
$$


and calculate the form of the potential as a function of $f$ and $g$. With $g=1$ this reduces to (5.4) so we would like to see how the potential changes as $g$ changes. Plugging (5.15) into (2.12), we have the following potential in the mass-deformed theory,

$$
V_{\mathrm{m}}(f, g)=\left(\frac{k}{2 \pi}\right)^{2} \mu^{3}\left[|f|^{2}\left(|g|^{2}-1\right)^{2}+|g|^{2}\left(|f|^{2}-1\right)^{2}\right]
$$

Then $V_{\mathrm{m}}$ vanishes at $f=g=0$ and $|f|=|g|=1$ as it should be. From this potential we get the quartic potential $\left(|f|^{2}-1\right)^{2}$ with $g=1$ which is the potential appearing in Maxwell-Higgs theory. Note that $f=0, g=1$ is not a local maximum of the potential since $V_{\mathrm{m}}(f=0, g) \sim|g|^{2}$. One may wonder how the configuration does not roll down to the origin. This is due to the special nature of the Gauss law in Chern-Simons gauge theory, namely the magnetic field is proportional to the charge density (3.9). Replacing $D_{0} Y^{2}$ by the magnetic field in the energy expression, we obtain an effective potential term $|B / g|^{2}$ which acts as a barrier at the origin $(g \rightarrow 0)$. This can be interpreted as a centrifugal term inversely proportional to $1 / g^{2}$ due to the rotation in $Y^{2}$ plane.

Along the direction $f=g$, (5.16) becomes the sextic potential $|f|^{2}\left(|f|^{2}-1\right)^{2}$ which appears in U(1) self-dual Chern-Simons matter system [10]. It turns out that this direction corresponds to a less supersymmetric BPS case and will be reported elsewhere [34.

\section{$5.2 \mathrm{U}(N) \times \mathrm{U}(N)$}

As in the previous subsection we start with the constraint $\beta_{4}^{43}=\mu Y^{3}$ and $\beta_{3}^{34}=\mu Y^{4}$. Solutions of these equations have already been considered in the end of section 2, For the irreducible solution (2.39), it is easy to show that the other constraints produce only a trivial solution $Y^{1}=Y^{2}=0$. Therefore to obtain nontrivial solutions we should consider reducible cases. Since there are many different possibilities, here we will analyze only some representative cases.

First note that the vorticity is carried by the field $Y^{1}$ and the other scalars constrain the degrees of freedom of $Y^{1}$ through constraint equations in (3.8). Then in order to find nontrivial solutions it is desirable to assume that $Y^{2}, Y^{3}, Y^{4}$ take simple forms. However, we cannot put all of these to be zero because the magnetic field would vanish in that case. Furthermore, if one of $Y^{3}, Y^{4}$ vanishes, the other should also vanish from the last two constraints in the third line of (3.8). Considering all these, it is natural to set $Y^{3}=Y^{4}=0$ while $Y^{2} \neq 0$. Then we are left with only one constraint $\beta_{2}^{21}+\mu Y^{1}=0$ as in the previous subsection.

An interesting nontrivial solution is obtained with the ansatz which generalizes (5.4) with $a=0$ to a block matrix form,

$$
Y^{1}=\sqrt{\frac{k \mu}{2 \pi}}\left(\begin{array}{cc}
0_{N_{1} \times N_{1}} & F_{N_{1} \times N_{2}} \\
0_{N_{2} \times N_{1}} & 0_{N_{2} \times N_{2}}
\end{array}\right), \quad Y^{2}=\sqrt{\frac{k \mu}{2 \pi}}\left(\begin{array}{cc}
0_{N_{1} \times N_{1}} & 0_{N_{1} \times N_{2}} \\
0_{N_{2} \times N_{1}} & G_{N_{2} \times N_{2}}
\end{array}\right),
$$

where $N_{1}, N_{2}$ are positive integers satisfying $N_{1}+N_{2}=N$ and the subscript denotes the dimensionality of each block which we omit from now on. We also only consider the case $N_{1} \geq N_{2}$ 
for simplicity. With the help of gauge symmetry, $G$ can be chosen to be a diagonal matrix with real nonnegative entries. Solving the constraint, we find $F=F G^{\dagger} G$ which implies $G$ is the $N_{2}$-dimensional identity matrix. Then the magnetic field is given by

$$
B=\hat{B}=-2 s \mu^{2}\left(\begin{array}{cc}
0 & 0 \\
0 & I-F^{\dagger} F
\end{array}\right)
$$

and up to a gauge we can write

$$
A_{i}=\hat{A}_{i}=\left(\begin{array}{cc}
0 & 0 \\
0 & \tilde{A}_{i}
\end{array}\right) .
$$

The only remaining equation is (3.5) and it becomes

$$
\left(D_{1}+i s D_{2}\right) F^{\dagger}=0
$$

where $D_{i} F^{\dagger}=\partial_{i} F^{\dagger}+i \tilde{A}_{i} F^{\dagger}$. Together with (5.18) this forms the nonabelian vortex equation in $\mathrm{U}\left(N_{2}\right)$ Yang-Mills theory with $N_{1}$ fundamental scalar fields and has been studied extensively [35, 36]. If $N=2$ and $N_{1}=N_{2}=1$, it reduces to the abelian vortex equation obtained in the previous subsection. For this configuration, we have $j^{0}=J_{12}^{0}=\frac{k}{2 \pi} B$ and obtain the energy as

$$
E=\left|\frac{k \mu}{2 \pi} \operatorname{tr} \int d^{2} x B\right|=n k \mu,
$$

which is the generalization of (5.13).

The ansatz (5.17) with $G=I$ may be considered as a solution to $\beta_{2}^{21}+\mu Y^{1}=0$ with maximal degeneracy. The irreducible nondegenerate ansatz similar to (2.39) is

$$
Y_{m n}^{1}=\delta_{m+1, n} \sqrt{\frac{k \mu}{2 \pi}} f_{m}, \quad Y_{m n}^{2}=\delta_{m n} \sqrt{\frac{k \mu}{2 \pi}} a_{m}
$$

where

$$
a_{m}=\sqrt{a_{1}^{2}+m-1} .
$$

Here $a_{1}$ is a nonnegative constant and $f_{1}, \ldots, f_{N-1}$ are functions to be determined. The irreducible vacuum would be obtained for $a_{1}=0$ and $f_{n}=\sqrt{N-n}$. With this ansatz the magnetic field becomes a diagonal matrix given by

$$
B_{m n}=\hat{B}_{m n}=-2 s \mu^{2} a_{m}^{2}\left(\left|f_{m}\right|^{2}-\left|f_{m-1}\right|^{2}+1\right) \delta_{m n}
$$

where $f_{0}=f_{N}=0$. Eliminating the gauge fields from (3.5), we obtain $N-1$ coupled differential equations,

$$
\partial \bar{\partial} \ln \left|f_{m}\right|^{2}=-\mu^{2}\left[a_{m}^{2}\left|f_{m-1}\right|^{2}-\left(a_{m}^{2}+a_{m+1}^{2}\right)\left|f_{m}\right|^{2}+a_{m+1}^{2}\left|f_{m+1}\right|^{2}+1\right]
$$

This type of coupled equations has appeared in $\mathrm{U}(1)^{N-1}$ gauge theories with $N-1$ Higgs fields which couple to the gauge fields [37]. 
As in $\mathrm{U}(2) \times \mathrm{U}(2)$ case, the solutions of (5.25) do not necessarily have finite energy. For the finite energy in (3.14), the trace of the magnetic field should vanish in the asymptotic limit $r \rightarrow \infty$. It is not difficult to find that the condition is consistent with the asymptotic behavior obtained from (5.25) only when the constant $a_{1}$ vanishes. Otherwise we would have infinite energy configurations with background of a constant magnetic field as discussed in the previous subsection.

From now on we consider only the case $a_{1}=0$ for which (5.25) reduces to

$$
\partial \bar{\partial} \ln \left|f_{m}\right|^{2}=-\mu^{2}\left[(m-1)\left|f_{m-1}\right|^{2}-(2 m-1)\left|f_{m}\right|^{2}+m\left|f_{m+1}\right|^{2}+1\right] .
$$

The asymptotic value of $\left|f_{m}\right|$ 's are determined by requiring that the right hand side of (5.26) vanish,

$$
\left|f_{m}\right| \rightarrow \sqrt{N-m}, \quad r \rightarrow \infty .
$$

Note that this is nothing but the irreducible vacuum values as it should be. An obvious solution of (5.26) is obtained with the ansatz

$$
f_{m}=\sqrt{N-m} f
$$

which is consistent with (5.27). Then (5.26) reduces to a single equation,

$$
\partial \bar{\partial} \ln |f|^{2}=-\mu^{2}\left(1-|f|^{2}\right),
$$

which is again the Maxwell-Higgs vortex equation which generalizes the result of $\mathrm{U}(2) \times \mathrm{U}(2)$ case in the previous subsection.

The ansatz (5.28) assumes that all the components $f_{m}$ 's have the same functional form and, in particular, the same vorticity. There are however more general solutions for which $f_{m}$ 's carry different vorticities [37]. Let $n_{m}$ be the vorticity of $f_{m}$. Then the energy can be calculated by taking the trace of the magnetic field,

$$
E=\left|\frac{k \mu}{2 \pi} \operatorname{tr} \int d^{2} x B\right|=k \mu\left|\sum_{l=2}^{N} \sum_{m=1}^{l-1} n_{m}\right| .
$$

If $n_{m}=n$ are the same for all $m$, the energy reduces to $E=n k \mu N(N-1) / 2$.

Other than the cases considered above, we have tried some other ansatz on which we briefly comment here. For $\mathrm{U}(3) \times \mathrm{U}(3)$, we worked out the equations in the most general way including reducible cases. In most cases the result is essentially some embedding of $\mathrm{U}(2) \times \mathrm{U}(2)$ case. When $Y^{2}=0$ while $Y^{3}, Y^{4}$ are not zero, Liouville-type equation can also be obtained as in the previous section. For $N>3$, we did not fully analyzed the constraints but it seems that most cases fall into one of those considered here. 


\section{Summary and Outlook}

In this paper, we investigated vortex-type half-BPS equations in the ABJM theory with or without mass deformation We obtained the energy bound (3.14) which is proportional to the massdeformation parameter. We also showed that the D-term deformation and the F-term deformation are the same as the mass deformation preserving maximal $\mathcal{N}=6$ supersymmetry.

For the undeformed ABJM theory, we solved all the constraint equations in the BPS equations. The resulting equation is shown to be the half-BPS equation in supersymmetric Yang-Mills theory. It has no finite energy regular solution.

In the mass-deformed theory, we showed that the BPS equations for $\mathrm{U}(2) \times \mathrm{U}(2)$ case reduce to the vortex equation appearing in Maxwell-Higgs theory which is known to have multi-vortex solutions. We obtained pure vortex solutions with the energy given by its vorticity as well as vortices in the constant background of magnetic field. We explored the origin of Maxwell-Higgs vortex in the Chern-Simons gauge theory. For $\mathrm{U}(N) \times \mathrm{U}(N)$ case with $N>2$, we obtained the nonabelian vortex equation of Yang-Mills-Higgs theory and also more general equations. It would be interesting to study the moduli space of these solutions.

There are many issues not addressed in this paper. It is straightforward to extend our analysis to the cases with less supersymmetry. A notable case among them is the $\mathcal{N}=1$ BPS equation which turns out to reduce to the vortex equation in U(1) Chern-Simons-Higgs system [10]. This has been also considered in [19] in the context of F-term deformation.

Since the ABJM theory is defined on a $\mathbb{Z}_{k}$ orbifold with the action $Y^{A} \rightarrow e^{2 \pi i / k} Y^{A}$, one may explore the possibility of configurations having fractional vorticity with phase dependence of the form $Y^{A} \sim e^{i \theta / k}$. It can be shown that this is possible in less supersymmetric solutions such as $\mathcal{N}=1$ case [34].

In this paper, we considered the theory purely from the viewpoint of a field theory and did not attempt to interpret the solutions in the context of M-theory. We would like to investigate these issues in the forthcoming publication [34].

\section{Acknowledgments}

The authors thank Ki-Myeong Lee and Sungjay Lee for useful discussions. H. N. thanks Masato Arai, Giacomo Marmorini and Muneto Nitta for helpful discussions. This work was supported by Astrophysical Research Center for the Structure and Evolution of the Cosmos (ARCSEC)) (O.K.) and grant No. R01-2006-000-10965-0 from the Basic Research Program through the Korea Science \& Engineering Foundation (Y.K.,H.N.). This work was also supported by the Science Research Center Program of the Korea Science and Engineering Foundation through the Center for Quantum Spacetime (CQUeST) with grant number R11-2005-021 and the World Class University grant R32-2008-000-10130-0 (C.K.). 


\section{References}

[1] For a review, see P. K. Townsend, "Four lectures on M-theory," arXiv:hep-th/9612121.

[2] J. Bagger and N. Lambert, "Modeling multiple M2's," Phys. Rev. D 75, 045020 (2007) arXiv:hep-th/0611108; "Gauge Symmetry and Supersymmetry of Multiple M2-Branes," Phys. Rev. D 77, 065008 (2008) [arXiv:0711.0955 [hep-th]]; "Comments On Multiple M2branes," JHEP 0802, 105 (2008) [arXiv:0712.3738 [hep-th]].

[3] A. Gustavsson, "Algebraic structures on parallel M2-branes," Nucl. Phys. B 811, 66 (2009) arXiv:0709.1260 [hep-th]].

[4] O. Aharony, O. Bergman, D. L. Jafferis and J. Maldacena, "N=6 superconformal ChernSimons-matter theories, M2-branes and their gravity duals," JHEP 0810, 091 (2008) arXiv:0806.1218 [hep-th]].

[5] A. Basu and J. A. Harvey, "The M2-M5 brane system and a generalized Nahm's equation," Nucl. Phys. B 713, 136 (2005) arXiv:hep-th/0412310.

[6] K. Hosomichi, K. M. Lee and S. Lee, "Mass-Deformed Bagger-Lambert Theory and its BPS Objects," Phys. Rev. D 78, 066015 (2008) [arXiv:0804.2519 [hep-th]].

[7] C. Krishnan and C. Maccaferri, "Membranes on Calibrations," JHEP 0807, 005 (2008) arXiv:0805.3125 [hep-th]].

[8] G. Bonelli, A. Tanzini and M. Zabzine, Phys. Lett. B 672 (2009) 390 arXiv:0807.5113 [hep-th]].

[9] S. Terashima, "On M5-branes in N=6 Membrane Action," JHEP 0808, 080 (2008) arXiv:0807.0197 [hep-th]];

K. Hanaki and H. Lin, "M2-M5 Systems in N=6 Chern-Simons Theory," JHEP 0809, 067 (2008) arXiv:0807.2074 [hep-th]].

[10] J. Hong, Y. Kim and P. Y. Pac, "On The Multivortex Solutions Of The Abelian ChernSimons-Higgs Theory," Phys. Rev. Lett. 64, 2230 (1990);

R. Jackiw and E. J. Weinberg, "Selfdual Chern-Simons Vortices," Phys. Rev. Lett. 64, 2234 (1990).

[11] C. Lee, K. M. Lee and E. J. Weinberg, "Supersymmetry And Selfdual Chern-Simons Systems," Phys. Lett. B 243, 105 (1990).

[12] R. Jackiw, K. M. Lee and E. J. Weinberg, "Selfdual Chern-Simons solitons," Phys. Rev. D 42, 3488 (1990). 
[13] C. Kim, "Selfdual vortices in the generalized Abelian Higgs model with independent ChernSimons interaction," Phys. Rev. D 47, 673 (1993) arXiv:hep-th/9209110].

[14] L. F. Cugliandolo, G. Lozano, M. V. Manias and F. A. Schaposnik, "Bogomolny equations for nonAbelian Chern-Simons Higgs theories," Mod. Phys. Lett. A 6, 479 (1991); K. M. Lee, "Selfdual nonabelian Chern-Simons solitons," Phys. Rev. Lett. 66, 553 (1991); "Relativistic nonAbelian selfdual Chern-Simons systems," Phys. Lett. B 255, 381 (1991).

[15] J. Gomis, D. Rodriguez-Gomez, M. Van Raamsdonk and H. Verlinde, "A Massive Study of M2-brane Proposals," JHEP 0809, 113 (2008) [arXiv:0807.1074 [hep-th]].

[16] I. Jeon, J. Kim, N. Kim, S. W. Kim and J. H. Park, "Classification of the BPS states in Bagger-Lambert Theory," JHEP 0807, 056 (2008) [arXiv:0805.3236 [hep-th]].

[17] I. Jeon, J. Kim, B. H. Lee, J. H. Park and N. Kim, "M-brane bound states and the supersymmetry of BPS solutions in the Bagger-Lambert theory," arXiv:0809.0856 [hep-th].

[18] J. Kim and B. H. Lee, "Abelian Vortex in Bagger-Lambert-Gustavsson Theory," JHEP 0901, 001 (2009) [arXiv:0810.3091 [hep-th]].

[19] M. Arai, C. Montonen and S. Sasaki, "Vortices, Q-balls and Domain Walls on Dielectric M2-branes," JHEP 0903, 119 (2009) arXiv:0812.4437 [hep-th]].

[20] N. Drukker, J. Gomis and D. Young, "Vortex Loop Operators, M2-branes and Holography," JHEP 0903, 004 (2009) [arXiv:0810.4344 [hep-th]].

[21] S. Mukhi and C. Papageorgakis, "M2 to D2," JHEP 0805, 085 (2008) arXiv:0803.3218 [hep-th]].

[22] Y. Pang and T. Wang, "From N M2's to N D2's," Phys. Rev. D 78, 125007 (2008) arXiv:0807.1444 [hep-th]].

[23] D. Gaiotto, S. Giombi and X. Yin, "Spin Chains in N=6 Superconformal Chern-SimonsMatter Theory," JHEP 0904, 066 (2009) arXiv:0806.4589 [hep-th]].

[24] M. A. Bandres, A. E. Lipstein and J. H. Schwarz, "Studies of the ABJM Theory in a Formulation with Manifest SU(4) R-Symmetry," JHEP 0809, 027 (2008) arXiv:0807.0880 [hep-th]].

[25] K. Hosomichi, K. M. Lee, S. Lee, S. Lee, J. Park and P. Yi, "A Nonperturbative Test of M2-Brane Theory," JHEP 0811, 058 (2008) arXiv:0809.1771 [hep-th]].

[26] K. Hosomichi, K. M. Lee, S. Lee, S. Lee and J. Park, "N=5,6 Superconformal Chern-Simons Theories and M2-branes on Orbifolds," JHEP 0809, 002 (2008) arXiv:0806.4977 [hep-th]]. 
[27] M. Benna, I. Klebanov, T. Klose and M. Smedback, "Superconformal Chern-Simons Theories and $\mathrm{AdS}_{4} / \mathrm{CFT}_{3}$ Correspondence," JHEP 0809, 072 (2008) arXiv:0806.1519 [hep-th]].

[28] K. Hosomichi, K. Lee, S. Lee, private communication.

[29] M. M. Sheikh-Jabbari and J. Simon, "On Half-BPS States of the ABJM Theory," arXiv:0904.4605 [hep-th].

[30] A. M. Low, "N=6 Membrane Worldvolume Superalgebra," JHEP 0904 (2009) 105 arXiv:0903.0988 [hep-th]].

[31] B. Grossman, "Hierarchy of soliton solutions to the gauged nonlinear Schrodinger equation on the plane," Phys. Rev. Lett. 65, 3230 (1990).

[32] G. V. Dunne, R. Jackiw, S. Y. Pi and C. A. Trugenberger, "Selfdual Chern-Simons solitons and two-dimensional nonlinear equations," Phys. Rev. D 43, 1332 (1991) [Erratum-ibid. D 45, 3012 (1992)].

[33] C. H. Taubes, "Arbitrary N: Vortex Solutions To The First Order Landau-Ginzburg Equations," Commun. Math. Phys. 72, 277 (1980).

[34] G. Go, C. Kim, Y. Kim, O-K. Kwon, H. Nakajima, in preparation.

[35] A. Hanany and D. Tong, "Vortices, instantons and branes," JHEP 0307, 037 (2003) arXiv:hep-th/0306150.

[36] M. Eto, Y. Isozumi, M. Nitta, K. Ohashi and N. Sakai, "Solitons in the Higgs phase: The moduli matrix approach," J. Phys. A 39 (2006) R315 arXiv:hep-th/0602170.

[37] C. Kim, C. Lee, P. Ko, B. H. Lee and H. Min, "Schrodinger fields on the plane with U(1)**N Chern-Simons interactions and generalized selfdual solitons," Phys. Rev. D 48, 1821 (1993) arXiv:hep-th/9303131. 